\title{
Discounting in an Uncertain World - Disentangling the Debate on the Weitzman-Gollier Puzzle
}

\author{
Wolfgang Buchholz
}

\author{
CESIFO WORKING PAPER NO. 4967 \\ CATEgORY 10: ENERGY AND ClimAte ECONOMICS \\ SEPTEMBER 2014
}

An electronic version of the paper may be downloaded

- from the SSRN website:

- from the RePEc website:

- from the CESifo website:

WWw.SSRN.com

www.RePEc.org

www.CESifo-group.org/wp

\section{CESifo}




\title{
Discounting in an Uncertain World - Disentangling the Debate on the Weitzman-Gollier Puzzle
}

\begin{abstract}
Under conditions of risk it makes a difference whether the discount rate is determined as an expected present or as an expected future value. This difference which is dubbed as the Weitzman-Gollier puzzle has stimulated an intensive discussion which, however, is somewhat confusing. In this paper we try to provide some clarification by separating the different topics of the debate. In particular we give an intuitive explanation for the divergence between the outcomes of the present and the future value approach. Moreover, we show that a declining time patter of the discount rate which is the major implication of Weitzman's present value approach can also be obtained in many other and more appealing ways.
\end{abstract}

JEL-Code: D610, D810, D990, G110.

Keywords: cost-benefit analysis, discounting, risk, Weitzman-Gollier Puzzle.

Wolfgang Buchholz

University of Regensburg

Universitäts Str. 31

Germany - 93040 Regensburg

wolfgang.buchholz@wiwi.uni-regensburg.de

August 28, 2014 


\section{Introduction}

Choosing the appropriate social discount rate has been a controversial issue in economics for a long time which, in the course of the climate change debate, received even more attention in the past few years (see e.g. Stern, 2007, or IPCC, 2014). The deep conceptual questions which arise in this context already in the case of certainty are amplified when uncertainty is brought into play and evaluation is not only across time but also across states. Therefore, it does not come as a surprise that discounting under uncertainty has been a somewhat neglected issue in the literature on discounting.

Weitzman (1998), however, has examined the question of discounting under risk and provided a quite simple answer to it: In case of uncertain interest rates a discount rate should be used which declines over time and in the long run converges to its lowest possible level. Weitzman's analysis has been of remarkable importance not only for the theory of discounting but also for empirical applications of cost-benefit analysis over time (see Arrow et al., 2013, 2014). From an ethical perspective Weitzman's result is particularly attractive since lower discount rates give higher weight to the interest of future generations and thus make sustainable development more likely.

But Weitzman's approach did not remain uncontested. Gollier (2004) has challenged Weitzman's position by giving a decision-theoretic argument which in contrast entails discount rates increasing over time and converging to the highest possible level. This fundamental difference of proposals for discounting under uncertainty has stimulated an intensive debate and by now is known as the "WeitzmanGollier puzzle". Many authors have made attempts to "resolve" it (see e.g. Hepburn and Groom, 2007, Buchholz and Schumacher, 2008, Freeman, 2010, Traeger, 2013) and, in a well-received paper, also Weitzman and Gollier (2011) themselves have tried to "reconcile" their originally diverging positions (see also Weitzman, 2010, and Gollier, 2012).

Until now the debate on the Weitzman-Gollier puzzle does not seem to have come to an end and the "literature has not converged at this stage to a consensus in the profession about the efficiency of the (Weitzman net present value, W.B.) 
NPV rule ... with a decreasing term structure of interest rates." (Gollier, 2013, p.12). But even consensus about the true meaning of the puzzle seems to be lacking since at least three different questions have been related to the puzzle and are often mixed up:

- What explains the different outcomes from Weitzman's and Gollier's approaches and how can the difference be made intuitively plausible?

- Which one of the two approaches appears to be more suitable for the determination of long-term discount rates in case of risk?

- What reasons might in general justify declining discount rates (DDR) over time?

To clarify the discussion on the Weitzman-Gollier puzzle and to further the analysis of discounting under risk it will be shown in this note that

- at the theoretical level the puzzle can be solved insofar as there is a simple and intuitive explanation for the difference between present and future value discount rates.

- concerning the suitability of the two approaches to real decision problems empirical and normative questions have to be answered in advance.

- the central and w.r.t. applications most interesting question whether DDR is warranted can be answered in the affirmative in different ways which, however, are not directly related to the puzzle as such.

In order to make the arguments more succinct I will present them with help of 19 claims. After preparatory Claims 1 and 2 the Claims 3 - 8 will refer to the first question which, surprisingly, has been slightly neglected in the whole discussion on the puzzle. Claims 9 - 13 then deal with the second question and Claims 14-16 with the third question. Claims 17 - 19 finally put the question of appropriate discounting under uncertainty into a broader perspective.

\section{The Claims}

Claim 1: The starting point of the puzzle is the determination of certainty equivalents for discount rates 
- for risk-neutral decision-makers.

- for non-marginal investment projects without arbitrage considerations along efficient paths.

- in a two-period setting.

The assumptions in point 1 and 2 were underlying Weitzman's (1998) original paper (as well as the contribution of Pazner and Razin (1975) who observed the cause of the puzzle for the first time). The possibility to simplify the argument (as indicated in point 3 above), however, is not used in most contributions to the puzzle.

Claim 2: Under the conditions stated in Claim 1 the expected present and the expected future value of an investment always differ when productivities are uncertain. The reason for this difference is not quite obvious at first sight so that indeed a theoretical puzzle arises. Only an economically meaningful explanation of the difference between the two discount factors should be considered as a resolution to the puzzle.

Explanation: In period 1 an investment is made which (per Euro invested) yields the payoff $m_{i}$ in state $i$ in period 2, i.e. $r_{i}=m_{i}-1$ is the rate of return in state $i$. There are $n$ possible states, and probability of state $i=1, \ldots, n$ is denoted by $p_{i}$. Gollier's Future Value FV-discount factor then is

$$
D_{G}=\frac{1}{\sum_{i=1}^{n} p_{i} m_{i}}
$$

Weitzman's Present Value PV-discount factor instead is

$$
D_{W}=\sum_{i=1}^{n} \frac{p_{i}}{m_{i}} .
$$


In the case of certainty, i.e. if $n=1$ and thus $p_{1}=1$, both discount factors coincide: $D_{G}=D_{W}=\frac{1}{m_{1}}$. This common discount factor indicates the present value of 1 Euro received or spent tomorrow.

If, however, uncertainty prevails, i.e. $n>1$ and $m_{j} \neq m_{k}$ for at least two $j, k=1, \ldots, n$ the discount factors $D_{G}$ and $D_{W}$ will be different and $D_{W}>D_{G}$ holds. It is this observation which gives rise to the puzzle. To make this precise we calculate

$$
\frac{D_{W}}{D_{G}}=\sum_{i=1}^{n} p_{i}^{2}+\sum_{j<k}\left(\frac{m_{j}}{m_{k}}+\frac{m_{k}}{m_{j}}\right) p_{j} p_{k}=\sum_{j=1}^{n} p_{i}^{2}+\sum_{j<k} \frac{m_{j}^{2}+m_{k}^{2}}{m_{j} m_{k}} p_{j} p_{k}
$$

Since $\left(m_{j}-m_{k}\right)^{2} \geq 0$ implies $\frac{m_{j}^{2}+m_{k}^{2}}{m_{j} m_{k}} \geq 2$ for all $m_{j}$ and $m_{k}$ (with equality if and only if $m_{j}=m_{k}$ ) we get $D_{W}>D_{G}$ if at least two productivity parameters $m_{i}$ differ, i.e. if there is some uncertainty, $D_{W}=D_{G}$ results if and only if all $m_{i}{ }^{\prime} s$ are identical which brings us back to the certainty case.

In order to establish the relationship between this two-period analysis and the continuous-time models mostly used in the literature let the rates of transformation $m_{i}$ be time-dependent with $m_{i}(t)=e^{r_{t} t}$ for all points in time $t>0$ where $r_{i}$ is the discount rate in state $i=1, \ldots, n$ and $r_{1} \leq \ldots \leq r_{n}$ is assumed. Then as special versions of (1) and (2) we get

$$
\begin{aligned}
e^{-r_{G}(t) t}=D_{G}(t) & =\frac{1}{\sum_{i=1}^{n} p_{i} e^{r_{t} t}}=\frac{e^{-r_{n} t}}{p_{n}+\sum_{i=1}^{n-1} p_{i} e^{\left(r_{i}-r_{n}\right) t}} \\
e^{-r_{W}(t) t} & =D_{W}(t)=\sum_{i=1}^{n} p_{i} e^{-r_{i} t}=e^{-r_{i} t}\left(p_{1}+\sum_{i=2}^{n} p_{i} e^{\left(r_{i}-r_{i}\right) t}\right)
\end{aligned}
$$


Taking logarithm in ( $\left.1^{\prime}\right)$ and $\left(2^{\prime}\right)$ yields

- the FV-discount rate $r_{G}(t)=-\frac{1}{t} \ln D_{G}(t)$ which is increasing in $t$ and converges to the maximum interest rate $r_{n}$.

- the PV-discount rate $r_{W}(t)=-\frac{1}{t} \ln D_{W}(t)$ which is decreasing in $t$ and converges to the minimum interest rate $r_{1}$ so that a justification for DDR is obtained. But note that in Weitzman (1998) not the average discount rate as defined by $\left(2^{\prime}\right)$ is considered but the instantaneous discount rate at time $t$ which is $\widehat{r}_{W}(t) \quad \dot{D}_{W}(t) / D_{W}(t)$. But e.g. by applying l'Hopital's rule it follows that $\lim _{t \rightarrow \infty} r^{W}(t)=\lim _{t \rightarrow \infty} \widehat{r}^{W}(t) \quad r_{1}$. Moreover, Weitzman (1998) only gives a proof for the convergence for $\widehat{r}_{W}(t)$ but not for its monotonicity and thus for DDR along the entire time axis. A proof of this result follows from the Appendix.

Claim 3: The difference between FV- and the PV-discount factors crucially hinges on the distribution of the payoffs in the two periods. It is this dependency which basically explains the difference between the two discount factors and thus can be looked upon as the starting point for a resolution of the puzzle.

Explanation: Assume that level of investment $d_{i}$ in period 1 may be statedependent which gives payoffs $e_{i}=m_{i} d_{i}$ in state $i=1, \ldots, n$ of period 2 . We then consider the rate of transformation between the average payoffs in period 2 and average investments in period 1:

$$
D_{T}:=\frac{\sum_{i=1}^{n} p_{i} d_{i}}{\sum_{i=1}^{n} p_{i} e_{i}}=\frac{\sum_{i=1}^{n} p_{i} d_{i}}{\sum_{i=1}^{n} p_{i} m_{i} d_{i}}=\frac{\sum_{i=1}^{n} p_{i} \frac{e_{i}}{m_{i}}}{\sum_{i=1}^{n} p_{i} e_{i}} .
$$

Given the identities in (5) the expression for $D_{T}$ appearing 
- after the second sign of equality can be interpreted as a generalized version of the FV-discount factor (with a distribution of payoffs in period 1 as the starting point).

- after the third sign of equality can be interpreted as a generalized version of the PV-discount factor (with a distribution of payoffs in period 2 as the starting point).

That - given $e_{i}=m_{i} d_{i}$ for all states $i=1, \ldots, n$ - both representations for the discount factor coincide is a main result in Gollier (2010) which, however, has been formulated for the case of marginal investments there.

Based on the identity (5) the central insight on which our explanation of the puzzle rests is that for a fixed expected value either in period 1 or period 2 the expected value in the other period depends on the distribution of the payoffs $d_{i}{ }^{\prime} s$ or $e_{i}^{\prime} s$ on which this fixed expected value is based. Hence, in case of uncertainty a unique rate of transformation which only depends on the expected values in both periods alone does not exist.

This impossibility result is easily confirmed by looking - in the $n$-dimensional space with the first period's payoffs $d_{i}^{\prime} s$ on the axis - at the $(n-1)$-dimensional hyperplanes which represent constant expected payoff values in the two periods. The gradient of any hyperplane for constant expected value of payoffs in period 1 is $\left(p_{1}, \ldots, p_{n}\right)$ while any hyperplanes for constant expected payoffs in period 2 has the gradient $\left(m_{1} p_{1}, \ldots, m_{n} p_{n}\right)$. Both gradients are parallel (so that the hyperplanes defined by them can coincide) if and only if $m_{1}=\ldots=m_{n}$, which leads to the case of certainty.

If, however, there is uncertainty some productivities $m_{i}$ the expression in (5) only gives a constant value for all $\left(d_{1}, \ldots, d_{n}\right)$ which lie on a $(n-2)$-dimensional hyperplane in which the $(n-1)$-dimensional hyperplanes with the gradients $\left(p_{1}, \ldots, p_{n}\right)$ and $\left(m_{1} p_{1}, \ldots, m_{n} p_{n}\right)$ intersect. If we assume that $m_{1} \leq \ldots \leq m_{n}$ these marginal rates of transformation vary between the lower bound $m_{1}$ and the upper bound $m_{n}$. 
In Figure 1, in which the first period payoff in state 1 is plotted on the horizontal axis and that in state 2 on the vertical axis, we illustrate this variation of transformation rates for the case $n=2$. Specifically we assume that $p_{1}=p_{2}=\frac{1}{2}$, and $m_{1}=1, m_{2}=2$ and that the expected value of payoffs in period 1 is equal to 1. Then the equal expected payoff line for period 1 is the (solid) straight line with slope -1 which cuts the axis in $A=(2,0)$ and $B=(0,2)$, respectively.

\section{Figure 1}

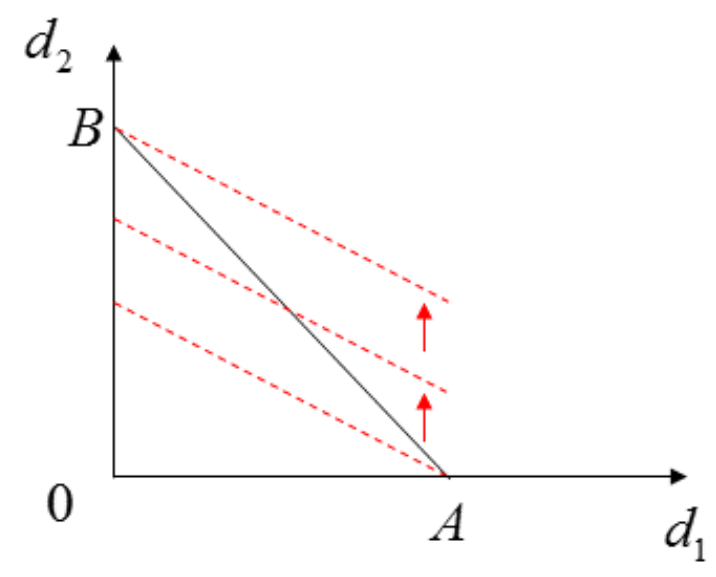

The loci of equal expected payoff in period 2 in contrast are the (hatched) straight lines with slope $-\frac{1}{2}$. The point of intersection ("0-dimensional hyperplane") of any such line with the straight line $A B$ indicates a certain level of the rate of transformation between average payoffs in period 1 and period 2 which - depending on $d_{1}$ - is $D_{T}\left(d_{1}\right)=2-\frac{d_{1}}{2}$ and thus ranges between 1 (in point $A$ ) and 2 (in point $B$ ).

Claim 4: The resolution of the paradox proposed by Weitzman and Gollier (2010) themselves also can be traced back to the generalized discount factor $D_{T}$. But in their attempt at reconciling their positions Weitzman and Gollier make use of the equivalence result provided by eq. (5) in a way which completely differs from the explanation of Claim 3 and does not seem overly convincing. 
Explanation: The approach chosen by Weitzman and Gollier (2010) is to adapt the probabilities of each state $i=1, \ldots, n$ to

$$
\begin{aligned}
& q_{i}^{G}=\frac{d_{i}}{\sum_{j=1}^{n} p_{j} d_{j}} p_{i} \\
& q_{i}^{W}=\frac{e_{i}}{\sum_{j=1}^{n} p_{j} e_{j}} p_{i} .
\end{aligned}
$$

A straightforward transformation then gives

$$
D_{T}=\frac{1}{\sum_{i=1}^{n} q_{i}^{G} m_{i}}=\sum_{i=1}^{n} \frac{q_{i}^{W}}{m_{i}} .
$$

so that for these adjusted probabilities the expressions for $D_{G}$ and $D_{W}$ formally yield the same discount factor. This procedure of calibrating the probabilities, however, is not motivated by any decision-theoretic argument and thus appears to be rather ad hoc. "This adjustment (of probabilities, W.B.) ... is elegant ..., however, it makes it hard to see the economic intuition underlying the adjustment" (Traeger, 2013, p. 579).

Instead of making a change of probabilities we will take another route to obtain the Weitzman and the Gollier discount factor as special versions of $D_{T}$ and assume specific payoff distributions for the two periods.

Claim 5: Starting with the general discount factor $D_{T}$ Gollier's discount factor $D_{G}$ is obtained by fixing a single payoff in period 1 while Weitzman's discount factor $D_{W}$ is obtained from $D_{T}$ by fixing a single payoff in period 2 . 
Explanation: Given $e_{i}=m_{i} d_{i}$ for all $i=1, \ldots, n$ it directly follows from (1), (2) and (5) that for the original "true" probabilities $\left(p_{1}, \ldots, p_{n}\right)$

- $D_{T}=D_{G}$ holds if the level of investment in period 1 is constant in all states of nature, i.e. $d_{i}=d$ for all $i=1, \ldots, n$.

- $D_{T}=D_{W}$ holds if the payoff of the risky project in period 2 is kept constant in all states of nature, i.e. $e_{i}=e$ for all $i=1, \ldots, n$.

Claim 6: From our explanation of the puzzle it moreover follows that for fixed probabilities $D_{G}$ and $D_{W}$ become identical when

- the two periods are interchanged and

- each rate of transformation $m_{i}$ between the periods is changed to its reciprocal $\frac{1}{m_{i}}$ for all $i=1, \ldots, n$.

In this sense the Weitzman-Gollier puzzle is indeed related to a "reversal of time and productivities" (Szekeres, 2014) which gives another interpretation of the puzzle.

Claim 7: From the perspective of Claim 5 the Gollier and the Weitzman discount factors give answers to two questions which only at first sight appear to be identical

- Gollier's question leading to $D_{G}$ : How many Euros have to be invested in period 1 to get 1 Euro on average in period 2?

- Weitzman's question leading to $D_{W}$ : How many Euros have to be invested on average in period 1 to get 1 Euro (for sure) in period 2?

In the case of risk both questions are different so that a priori identical answers cannot be expected. This is clearly seen by a simple example in which $n=2$, $p_{1}=p_{2}=\frac{1}{2}, m_{1}=\frac{1}{1000}$ and $m_{2}=1000$. Then we have 
- $D_{G}=\frac{1}{\frac{1}{2}\left(\frac{1}{1000}+1000\right)} \approx \frac{1}{500}$, i.e. the rate of transformation becomes very small: Due to the very high productivity in state 2 only a very small investment is required to get an average payoff equal to 1 in period 2 as it has to be attained in the Gollier scenario.

- $D_{W}=\frac{1}{2}\left(\frac{1}{\frac{1}{1000}}+\frac{1}{1000}\right) \approx 500$, i.e. the rate of transformation becomes very high: Due to the very low productivity in state 1 a very high investment is needed to get a sure payoff equal to 1 in period 2 as it has to be attained in the Weitzman scenario.

This elementary example already shows that in the Gollier case it is the state with the high productivity which dominates evaluation while in the Weitzman case it is the state with the low productivity which dominates. From the example it becomes also clear that in the case with two states $D_{G}$ goes to zero if $m_{1}$ is fixed and $m_{2}$ goes to infinity while $D_{W}$ goes to infinity if $m_{2}$ is fixed and $m_{1}$ goes to zero.

Claim 8: In line with Claim 7 the Weitzman and the Gollier approach capture completely different decision situations:

- The Gollier scenario rests upon the standard two-period model in which payments in the present ( $=$ investment costs in period 1$)$ are certain and payments in the future (= investment returns in period 2 ) are uncertain.

- The Weitzman scenario in fact presupposes a less conventional three-period model. In the new present (= period 0 ) investments with stochastic returns are assessed which have to be made in the near future (= period 1$)$ to provide a sure target payoff in the more distant future (= period 2 ). This interpretation was also briefly indicated by Weitzman (1998, p. 204) himself in one of the few sentences in which he motivates his discounting device: "Suppose that an investment with distant-future consequences must be made now, before we know what the relevant scenario will be." 
Claim 9: When a choice between Weitzman's and Gollier's discounting criterion is to be made the particular features of the situation in which the criteria should be applied have to be taken into account. This clearly is not a theoretical but an empirical matter. In any case Weitzman's discounting device should not - as recently in Arrow et al. (2013) - be used without a reflection of the empirical conditions under which the decision is made.

Claim 10: Notwithstanding the fact that the choice of the discount rate is situation-dependent it is legitimate to judge the empirical relevance of each approach at a general level. In this context it seems fair to say that Gollier's approach is more in line with standard expected utility models than Weitzman's: "Risk" and "future" are considered as almost synonymous which is clearly reflected by FV-discounting. In the case of PV-discounting, however, an unorthodox and rather intricate decision situation is assumed in which - as seen from the viewpoint of period 2 where investment returns materialize - the risk lies in the past. From this viewpoint Weitzman's PV-approach may indeed be deemed "less theoretically elegant" (Arrow et al., 2014, p. 161).

Claim 11: This prima facie superiority of FV-approach does not preclude that decision problems exist for which the PV-method is more suitable. So Weitzman's approach might not only fit better for a description of hedging activities on financial markets but also for some decisions in environmental economics.

Explanation: Coping with climate change in the long run requires ecologically friendly technological innovations. So decisions between paths of technological development have to be made today (= period 0 ). The true costs of these green technologies (e.g. different types of renewable energies like solar or wind) are only revealed after some lapse of time, i.e. in the near future (= period 1). In period 1 then these technologies have to be implemented at a large scale to ensure that some fixed environmental target (= a certain maximum concentration of green- 
house gases in the atmosphere) is attained with safety in the more distant future (= period 2), to avoid catastrophic climate change.

From this perspective Weitzman's approach is clearly helpful to reflect a central concern of climate policy since it assumes a fixed target or a threshold in the future. In a certain sense it thus reflects sustainability concerns.

Note that even in such a situation the discount factor will depend on the length of the time intervals between period 1 and period 2 on the one hand and period 2 and period 3 on the other. This issue deserves some closer analysis.

Claim 12: At the conceptual level any serious conflict between the two approaches vanishes if one accepts from the beginning that "one size does not fit all" and that no "right" solution can be obtained on purely theoretical grounds. Hence also in this sense the debate on the puzzle indeed is "moot" as stated in Szekeres' (2014) harsh (and partly overdone) critique of Weitzman's argument.

Claim 13: But even if one in principle adopts a relativistic position w.r.t. the comparison between PV- and FV-discounting one cannot help stating that Weitzman in fact does not provide a "'basic reason' or a 'generic' argument" for using certaintyequivalent discount rates that decline over time" (Weitzman, 1998, p. 204) - as it has been his declared intention and as it has partly been accepted in the literature. E.g. Cropper et al. (2014) still regard Weitzman's PV-approach as an independent justification for DDR. Despite this objection Weitzman deserves much credit for putting DDR on the scientific agenda (what he has also done in other ways, e.g. by aggregating experts' views on the appropriate discount rate (Weitzman, 2001)).

Claim 14: Concerning the main outcome of Weitzman's approach, i.e. appropriateness of DDR, the debate about the puzzle only is of limited relevance: Discount rates which are falling over time can quite easily be generated, e.g. by assuming specific time pattern for productivities in the different states of the world - even in the case of risk neutrality and without having to abandon the conventional framework with sure payoffs in the present and risky payoffs in the future (as in Gollier's approach). Therefore DDR by no means comes up as an exception or surprise. 
Explanation: Assume that there are general time paths for productivities $\left(m_{1}(t), \ldots, m_{n}(t)\right)_{t \geq 0}$ for which $\sum_{i=1}^{n} p_{i} m_{i}(0)=1$ holds. The corresponding discount rate $r_{M}(t)=r_{G}(t)$ at any point in time $t>0$ is then determined from (1) as

$$
r_{M}(t)=\frac{1}{t} \ln \left(\sum_{i=1}^{n} p_{i} m_{i}(t)\right)
$$

This discount factor is falling in $t$ if productivities $m_{i}(t)$ are increasing in $t$ but their growth is relatively slow so that the decreasing effect of the function $\frac{1}{t}$ dominates. This e.g. occurs if $n=2, p_{1}=p_{2}=\frac{1}{2}, m_{1}(t)=1+t \quad$ and $m_{2}(t)=1+2 t$ so that $r_{M}(t)=\frac{1}{t} \ln \left(1+\frac{3}{2} t\right)$ for all $t>0$. Obviously, $r_{M}(t)$ is declining and converges to zero if $t$ goes to infinity in this example.

Claim 15: DDR is also obtained in the otherwise unchanged model for nonmarginal investment under risk when the FV-criterion is adopted but the assumption of risk neutrality is dropped and a sufficiently high degree of risk aversion is supposed. This approach for justifying DDR seems to be closest to the framework in which Weitzman's and Gollier's arguments originally had been presented.

Explanation: Extending an argument of Buchholz and Schumacher (2008) we assume that $m_{i}(t)=e^{r_{i} t}$ for all $t>0$ and that the decision-maker is risk averse and has an isoelastic utility function $u_{\eta}(c)=\frac{c^{1-\eta}}{1-\eta}$ for which $\eta>1$ holds (and which thus is more risk averse than $\left.u_{1}(c)=\ln c\right)$. The FV-interest rate $r_{M}^{\eta}(t)$ at some point of time $t$ then is obtained from 


$$
\frac{1}{\left(e^{r_{M}^{\eta}(t) t}\right)^{1-\eta}}=\frac{1}{\sum_{i=1}^{n} p_{i}\left(e^{r_{i} t}\right)^{1-\eta}}
$$

which gives

$$
\begin{aligned}
r_{M}^{\eta}(t) & =\frac{1}{t(1-\eta)} \ln \sum_{i=1}^{n} p_{i} e^{r_{i}(1-\eta) t} \\
& =\frac{1}{t(1-\eta)} \ln \left(e^{r_{1}(1-\eta) t}\left(p_{1}+\sum_{i=2}^{n} p_{i} e^{\left(r_{i}-r_{1}\right)(1-\eta) t}\right)\right) \\
& =r_{1}+\frac{1}{t(1-\eta)} \ln \left(p_{1}+\sum_{i=2}^{1} p_{i} e^{\left(r_{i}-r_{1}\right)(1-\eta) t}\right) .
\end{aligned}
$$

The discount rate $r_{M}^{\eta}(t)$ converges to the minimum interest rate $r_{1}$ since $r_{i}-r_{1} \geq 0$ for all $i=2, \ldots, n$ combined with $1-\eta<0$ implies that $\lim _{t \rightarrow \infty} p_{i} e^{\left(r_{i}-r_{1}\right)(1-\eta) t}=0$ for all $i=2, \ldots, n$. By an additional argument it can also be demonstrated that the discount rate $r_{M}^{\eta}(t)$ is falling even for all $t>0$ (see the Appendix).

Claim 16: Assuming risk aversion and changing other assumptions and/or the model framework may also yield declining discount rates. So DDR follows when marginal investments along stochastic consumption growth path are considered e.g.

- if the "Weitzman channel" dominates the "Gollier channel" (as elaborated by Traeger, 2013).

- if in the CAPM-framework the beta of the project under consideration is not too large (see Gollier, 2014).

In these more complex models it is, however, not always ensured that DDR is obtained but depends on the specific assumptions. 
Explanation: While the Gollier channel refers to uncertainty of the productivity of investment the Weitzman channel works through the uncertain growth rate for baseline consumption on which the aggregate welfare effect of a marginal investment made today depends. This reflects the important insight that if, e.g., consumption in the future is much higher than today and - given a concave utility function - marginal utility thus is much smaller an investment with some given productivity will only result in a relatively small increase in future utility (as compared to the loss of utility today which is caused by financing the investment).

To make the influence which this Weitzman channel has on the time pattern of discount rates more precise we now assume that in the $n$ states of the world we move along consumption paths $c_{i}(t)=e^{g_{i} t}$ with some positive or negative growth rate $g_{i}(i=1, \ldots, n)$ and that productivity of a marginal investment made at time zero is $m(t)$ at time $t$. Then in the simple case without pure time discounting the aggregate change of expected utility which arises from this marginal investment is

$$
\sum_{i=1}^{n} p_{i} u^{\prime}\left(c_{i}(t)\right) m(t)-u^{\prime}(c(0))
$$

For the determination of the discount rate $r_{C}(t)$ in this scenario we have to look for that threshold level of productivity $m_{C}(t)=e^{r_{C}(t) t}$ for which the expression in (12) becomes zero: Any investment which materializes at time $t$ increases (decreases) total welfare if its productivity is higher (smaller) than $m_{C}(t)$.

As before we apply an isoelastic utility function with constant relative risk aversion $\eta$. Then marginal utility is $u_{\eta}^{\prime}(c)=c^{-\eta}$ and - as $c(0)=1$ - condition (12) boils down to

$$
e^{-r_{C}^{\eta}(t) t}=\sum_{i=1}^{n} p_{i} e^{-g_{i} \eta t}
$$

This gives 


$$
r_{C}^{\eta}(t)=-\frac{1}{t} \ln \sum_{i=1}^{n} p_{i} e^{-g_{i} \eta t}
$$

If probabilities are equal and $g_{i}=r_{i}$ for all $i=1, \ldots, n$ a comparison between (12) and (14) shows that

$$
r_{C}^{\eta}(t)=\eta r_{M}^{1+\eta}(t)
$$

Therefore, even though $r_{M}^{\eta}(t)$ and $r_{C}^{\eta}(t)$ originate from conceptually different scenarios they show a quite similar behavior. In case of $r_{C}^{\eta}(t)$, however, DDR is obtained for any $\eta>0$.

In the CAPM-framework a low value of beta indicates a low correlation between the productivity risk and the baseline consumption risk. In this case the "precautionary effect" which taken by itself would entail DDR dominates the "risk effect" which instead would lead to increasing discount rates over time (Gollier, 2014, p. 535; see also IPCC, 2014, pp. 34-35).

In Weitzman (1998, p. 204) the Weitzman channel, however, shines up only implicitly when it is said that "investment is 'small' relative to overall size of the world economy and its uncertainty is uncorrelated with the state of the world".

Claim 17: If one tries to develop criteria for long-term discounting in case of uncertainty and risk aversion more empirically oriented reflection about the structure of future risk is required. Especially in the climate change context it has to be explored more thoroughly how baseline consumption risk (i.e. uncertainty about future growth rates) might be affected by global warming, how this risk will be correlated with the productivity risk of greenhouse gas abatement investment and how all these risks may be correlated over time.

Claim 18: Risk is only one determinant for intertemporal evaluation among many others so that its specific implications for the long-run discount rate have to be 
qualified and compared with other determinants in the certainty case (which are relevant also in the certainty case).

Explanation: Looking at the Ramsey equation $r=\delta+g \eta$ under conditions of certainty (where $\delta$ is the pure rate of time discount, $g$ the growth rate of the economy and $\eta$ again is the elasticity of marginal utility) it becomes obvious that the interest rate will fall if the growth rate $g$ decreases over time while the other two parameters remain constant. (This phenomenon essentially also is underlying the explanation of Claim 14.) Decreasing growth rates, however, are the outcome of important economic models without having to invoke uncertainty. So in the classical Dasgupta-Heal-Solow model in which the input of an exhaustible natural resource is an argument of the production function it is a well-known fact (see e.g. Dasgupta and Heal, 1979, or Buchholz, 1982) that the growth rate and the consumption interest rate decline over time along any feasible path. Reviving the "secular stagnation" hypothesis some pessimism about the prospects for future growth has also become a relevant part of the recent literature on economic growth (see e.g. the controversial discussion in Baldwin and Teuling, 2014).

Claim 19: Due to the long-run consequences of global warming and the big structural change of the economy that an effective climate policy would necessitate, it might be inappropriate to focus the discussion on intertemporal valuation on discounting since this "... runs the risk of shoe-horning this parts of economics and ethics of climate change into a very narrow form" (Stern, 2013, p. 5) and may thus "divert attention from the big strategic issues" (p. 7).

Explanation: According to Stern (2013) discounting is a marginal concept whose application only makes sense for the assessment of small investment projects along predetermined growth paths but not for the selection among growth and emission paths. Especially this, however, seems to be the big challenge global climate change policy is faced with. The problems of intertemporal evaluation are amplified by the big uncertainties in the Knightian sense which are connected with any strategy to combat climate change at a global scale. Moreover, as future generations 
will be very much affected by global warming, intertemporal evaluation in the climate change context has to be concerned with distribution across generation and thus with issues of intergenerational equity. It seems questionable whether the usual discounting procedure is capable to handle these intricate ethical issues in an adequate way, especially when risk and uncertainty come into play.

\section{Conclusion}

The Weitzman and the Gollier precepts for determining the social discount rate are based on different assumptions about the situation and the objectives of the decision under risk so that neither Weitzman's nor Gollier's approach can simply be "wrong". In economics it is quite common that different model assumptions may lead to different and even contradictory conclusions. But from such a relativistic viewpoint there is no need at all for reconciling both approaches. As the different outcomes stemming from the two approaches can easily be explained even not much of a puzzle or even a paradox is left over.

Concerning the effects of intertemporal evaluation Weitzman's approach gives more weight to the future than Gollier's. In this sense it might be interpreted as a very specific application of the sustainability concept to specific long-run decisions under risk. This makes Weitzman's discounting device ethically appealing since it gives future generations some protection against the potentially detrimental effects of unfettered discounting at a constant rate.

But this important outcome can also be attained directly by some obvious modifications of the standard model since - without having to fall back upon less conventional decision situations - the DDR hypothesis can be substantiated by incorporating risk aversion into decision-theoretic models in several ways (and also in models without any risk). To put it directly: DDR can be justified by a lot of theoretical approaches which at the conceptual level seem to be much less problematic than Weitzman's.

Such modification of the theoretical framework is well-suited for the determination of social discount rates because it brings the decision situation much closer to reality. But for the sake of intellectual clarity such an adaptation of the model 
should not be confounded with the Weitzman-Gollier puzzle as such. Rather, the "change of the game" makes the puzzle obsolete from the start.

Acknowledgements: This paper was presented at the Workshop in Sustainability Economics: Intergenerational Equity and Efficiency under Uncertainty at Camp Reinsehlen, Germany, August $4-7,2014$. This revised version has benefitted a lot from the helpful comments of the participants. Financial support from the Germany Ministry of Education and research BMBF through the ECCUITY-project (Project No. 710703) is gratefully acknowledged. Many thanks also to Lisa Dippl and Michael Eichenseer for carefully checking the manuscript.

\section{Appendix: General Conditions for DDR}

Let in a general setting the marginal rate of substitution between payoffs in period 0 and payoffs in period $t$ be $\mu(t)$ for any $t \geq 0$ and assume that $\mu(0)=1$ and $\mu(t)<1$ for all $t>0$. The average discount rate at time $t>0$ then is given by

$$
r_{\Phi}(t)=-\frac{1}{t} \ln \mu(t)
$$

Step 1: $r_{\Phi}(t)$ is a decreasing function of $t$ if $\varphi(t):=-\ln \mu(t)$ is a concave function. This follows from the general fact that $\varphi(0)=0$ and $\varphi^{\prime \prime}(t)<0$ imply $\varphi^{\prime}(t) t<\varphi(t)$ and thus $\left(\frac{\varphi(t)}{t}\right)^{\prime}<0$

Step 2: The function $\varphi(t)$ is concave if

$$
\mu^{\prime}(t)^{2}-\mu^{\prime \prime}(t) \mu(t)<0
$$

holds for all $t$ which directly follows by taking the derivative of $\varphi^{\prime}(t)=-\frac{\mu^{\prime}(t)}{\mu(t)}$. 
Step 3: Assume that specifically

$$
\mu(t):=\sum_{i=1}^{n} p_{i} e^{-\rho_{i} t}
$$

with $\sum_{i=1}^{n} p_{i}=1, \rho_{i} \geq 0$ for all $i=1, \ldots, n$. Then condition A-3 is satisfied since

$$
\begin{aligned}
\mu^{\prime}(t)^{2}-\mu^{\prime \prime}(t) \mu(t) & =\sum_{\substack{j, k=1, \ldots, n \\
j \leq k}}\left(2 \rho_{j} \rho_{k}-\left(\rho_{j}^{2}+\rho_{k}^{2}\right)\right) p_{j} p_{k} e^{-\rho_{j} t} e^{-\rho_{k} t} \\
& =-\sum_{\substack{j, k=1, \ldots, n \\
j \leq k}}\left(\rho_{j}-\rho_{k}\right)^{2} p_{j} p_{k} e^{-\rho_{j} t} e^{-\rho_{k} t}<0
\end{aligned}
$$

if for some $j, k=1, \ldots, n$ with $j \neq k$ we have $\rho_{j} \neq \rho_{k}$.

\section{References}

Arrow, K. J. et al. (2013), Determining benefits and costs for future generations. Science 341, 349-350.

Arrow, K. J. et al. (2014), Should governments use a declining discount rate in project analysis? Review of Environmental Economics and Policy 8, 145-163.

Baldwin, R. and Teulings, C. (2014), Secular stagnation: Facts, causes and cures. A VoxEU.org eBook. Center of Economic Policy Research (CEPR), London UK.

Buchholz, W. (1982), On the possibility of economic growth with exhaustible resources, in: W. Eichhorn (ed.), Economic theory of natural resources. PhysicaVerlag, Würzburg - Wien, 295-300.

Buchholz, W. and Schumacher, J, (2008), Discounting the long-distant future: A simple explanation for the Weitzman-Gollier puzzle. CESifo Working Paper No. 2357.

Cropper, M. L. et al. (2014), Declining discount rates. American Economic Review: Papers \& Proceedings 104, 538-543. 
Dasgupta, P. and Heal, G. (1979), Economic theory and exhaustible resources. Cambridge University Press, Cambridge UK.

Freeman, M. C. (2010), Yes, we should discount the far distant future at its lowest possible rate: A resolution of the Weitzman-Gollier puzzle. The Open-Access, OpenAssessment E-Journal 4.

Gollier, Ch. (2004), Maximizing the expected net future value as an alternative strategy to gamma discounting. Finance Research Letters 3, 85-89.

Gollier, Ch. (2010), Expected net present value, expected net future value, and the Ramsey rule. Journal of Environmental Economics and Management 59, 142-148.

Gollier, Ch. (2012), Pricing the planet's future: The economics of discounting in an uncertain world. Princeton University Press, Princeton N.J.

Gollier, Ch. (2013), An economic justification to gamma discounting. Mimeo, Toulouse School of Economics.

Gollier, Ch. (2014), Discounting and growth. American Economic Review: Papers \& Proceedings 104, 534-537.

Gollier, Ch. and Weitzman, M. (2010), How should the distant future be discounted when discount rates are uncertain? Economics Letters 107, 150-153.

Hepburn, C. and Groom, B. (2007), Gamma discounting and expected net future value. Journal of Environmental Economics and Management 53, 99-109.

International Panel on Climate Change (IPCC) (2014), Climate change 2014: Mitigation of climate change. Chapter 3: Social, economic and ethical concepts and methods.

Pazner, E. A. and Razin, A. (1975), On expected present vs. expected future value. Journal of Finance 30, 875-877.

Stern, N. (2006), The economics of climate change: The Stern review. Cambridge University Press, Cambridge UK.

Stern, N. (2013), Ethics, equity and the economics of climate change. Paper 2: Economics and politics. Grantham Research Centre on Climate Change and the Environment, Working Paper No. 84b, London UK.

Szekeres, S. (2014), The Weitzman-Gollier puzzle is not a paradox but a mistake, and it is most likely moot. Mimeo. 
Traeger, Ch. (2013), Discounting under uncertainty: Disentangling the Weitzman and the Gollier effect. Journal of Environmental Economics and Management 66, 573-582.

Weitzman, M. (1998), Why the far-distant future should be discounted at its lowest possible rate. Journal of Environmental Economics and Management 36, 201-208.

Weitzman, M. (2001), Gamma discounting. American Economic Review 91, 260271. 